\title{
Metáforas de la individualidad moral y fundamentos de infoética*
}

\author{
EdUARDO DE BUSTOS \\ Dpto. de Lógica, Historia y Filosofía de la Ciencia. UNED, Madrid
}

\begin{abstract}
RESUMEN. La ética de la información propuesta por L. Floridi $(1999,2002)$ es la principal alternativa teórica que, por un lado, permite superar la polémica de la singularidad de la ética de los computadores y, por otro, dotar de un fundamento teórico general a dicha ética en un nivel ontológico. Su análisis crítico desvela los mecanismos cognitivos empleados en su constitución y, al mismo tiempo, pone en cuestión algunas de sus consecuencias, como la equiparación del mal moral con alguna forma de entropía.
\end{abstract}

Palabras clave: Ética de la Información, agencia moral, objeto informacional, proyección analógica.
ABSTRACT. Information Ethics, as proposed by L. Floridi $(1999,2002)$, is the main theoretic alternative that, in one side, allows to overcome the uniqueness debate about the computation ethics and, on the other, to give a general theoretic foundation to that kind of ethics at an ontological level. When it is critically analyzed, the analysis shows the cognitive resources used to build it up and, at the same time, it allows to critically consider some of its consequences, as the identification of moral evil with some kind of enthropy.

Key words: Information Ethics, moral agency, information object, analogical mapping.

\section{Introducción}

Las tecnologías de la comunicación e información (TIC) no sólo trasforman el acceso a la información, la producción de conocimiento y la vida social, sino que también, como consecuencia de ello, abren nuevos caminos a la reflexión ética, fuerzan a la consideración analítica y crítica de los nuevos desarrollos tecnológicos, de su impacto en la producción y distribución del conocimiento, y sobre la aparición de nuevos ámbitos de acciones o comportamientos éticos.

* La elaboración de este trabajo se ha visto beneficiada por la financiación otorgada por el Ministerio de Educación y Ciencia a los Proyectos de Investigación «La emergencia de las normas tecnocientíficas: desarrollo de una aproximación socio-cognitiva y aplicación a los casos de las nuevas tecnologías y la biomedicina» y «¿Artes del discurso vs. Teoría de la argumentación? Problemas y perspectivas de un marco teórico integrador de los estudios de la argumentación.» 
Una forma radical de abordar el problema de la ética y las TIC es mantener que esa clase de tecnologías dan lugar a una modificación sustancial del entorno en que vivimos, tanto en cuanto a una producción de nuevas realidades como a la modificación esencial de realidades previas. Es decir, se trata de una idea que no considera esas tecnologías como una extensión o ampliación de tecnologías previamente existentes, ni como su mera prolongación en el ámbito de su aplicación o de su generalización en la distribución de su uso. En ese sentido, su análisis ético no puede consistir en una simple aplicación analógica de análisis previos sobre otras realidades tecnológicas. Aunque esas realidades históricas hayan sido de suma importancia (la invención de la escritura, de la imprenta....) y hayan modificado el curso de la vida social y el progreso cultural, se diferencian claramente, al decir de esta posición, de las modificaciones que están produciendo las TIC. Y la diferencia consiste esencialmente en una reconstitución, que no una reconstrucción, del entorno humano, físico y social. Para decirlo con el término que utiliza L. Floridi (1999), la aparición de las TIC es la causa de una reontologización del medio en que se mueve el hombre. Esa modificación sustancial obliga a un doble esfuerzo de teorización: desde el punto de vista cognitivo, demanda un esfuerzo de conceptualización, de asimilación de las nuevas realidades y de integración de las nuevas formas de interacción con ellas. Desde el punto de vista práctico, requiere una reacomodación de nuestros valores a esas nuevas realidades y unas renovadas concepciones críticas de la aplicación de esos valores a las nuevas realidades tecnológicas, de nuevas formas de orientar nuestras acciones en ese entorno tan radicalmente novedoso.

\section{El debate de la singularidad de la infoética}

A la hora de analizar críticamente la presunta singularidad de la infoética, conviene tener en cuenta que las discusiones precedentes (Tavani, 2002) sobre la cuestión se han desarrollado jugando con dos planos: en primer lugar, el ámbito de la discusión fue el de si el uso de los computadores planteaba nuevas realidades o problemas éticos que no fueran abarcables mediante una aplicación directa de los principios normas o teorías éticas tradicionales. El foco del análisis era pues la materialidad del producto tecnológico, el ordenador, y el rango de las modificaciones que ese artefacto podía producir en ciertas formas de vida éticamente evaluables. En segundo lugar, en una especie de progresión semántica o argumentativa, el ámbito de la discusión se ha trasladado al concepto de información. Ya no se trata tanto del artefacto mismo del computador como de todas las nuevas tecnologías (computador incluido) que se pueden caracterizar por el uso de información. Es decir, la presunta singularidad de la infoética, frente a la más antigua ética de los computadores o compuética, reside en que tiene como objeto una realidad no reducible, al menos desde el punto de vista moral, a una realidad material. 
De acuerdo con el análisis de Tavani (2002), se dan dos posturas características frente a la cuestión de la singularidad de la ética de los computadores, o compuética: 1) los tradicionalistas, que mantendrían que no se dan novedades relevantes en cuanto a los crímenes o violaciones de la ley cometidos con participación de las TIC, de tal modo que «podemos utilizar las categorías morales tradicionales para analizar estas cuestiones y, sencillamente, lo que tenemos que hacer es aplicar las teorías éticas tradicionales a las cuestiones morales específicas relacionadas con los computadores y las TIC» (Tavani, 2002, 38). Así pues, para los tradicionalistas el esfuerzo cognitivo requerido por las posibles novedades de la presencia de las TIC en la vida social y moral es el de la aplicación, basada en procesos inferenciales de naturaleza analógica, de teorías y situaciones ya existentes en ámbitos en que las TIC no desempeñan ningún papel relevante. La importancia de las TIC es puramente circunstancial; enriquecen o hacen más compleja una situación moral, pero no modifican su naturaleza íntima.

Entre los no tradicionalistas, o proponentes de la singularidad de la ética computacional (singularistas) se dan diferentes matices (Tavani, 2002, 38). Por un lado, se mantiene que la ética computacional aborda nuevas cuestiones éticas, no existentes con anterioridad al advenimiento de las TIC. Otros en cambio sostienen que esas nuevas cuestiones son el efecto directo de la aparición de las TIC en el ámbito moral, y que no pueden ser afrontadas utilizando las teorías morales tradicionales: «este grupo de defensores de la singularidad sugiere que lo que es preciso es, o bien un nuevo tipo de teoría ética (como mínimo) o posiblemente todo un nuevo marco para la moralidad» (Tavani, 2002, 38),

Pero, como observa Tavani, la aparición de fenómenos nuevos con una dimensión moral no implica necesariamente la tesis de la singularidad de la ética computacional. El sentido relevante de 'único' o de 'singular' requiere que los fenómenos considerados no sólo sean nuevos, sino que de alguna forma exijan una reorganización de sistemas o recursos cognitivos previamente existentes. De acuerdo con Tavani, para considerar a un fenómeno X como 'singular' en el sentido relevante, « $\mathrm{X}$ ha de ser nuevo o novedoso en una forma que suponga un desafío o bien para a) nuestros esquemas existentes de categorización y clasificación de ese fenómeno en particular; o b) para nuestras formas previas de explicar y analizar X» (Tavani, 2002, 40). De acuerdo con el análisis de Tavani, se da una cierta ambigüedad en lo que los proponentes de la tesis de la singularidad mantienen: por un lado, parecen proponer una ampliación ontológica, en el sentido de una integración conceptual de las nuevas realidades (morales) ligadas a las TIC $\mathrm{y}$, por otro, una modificación radical de las teorías o marcos éticos para hacer frente a los problemas planteados por esas nuevas realidades.

Aunque, efectivamente, ambos aspectos son analíticamente diferentes, no es necesario que sean autónomos, esto es, que no puedan ser relacionados en términos causales. La revisión de nuestras teorías éticas puede estar motivada por la aparición de nuevas realidades, ya sean objetos o relaciones, que fuercen los límites conceptuales de esas teorías éticas. Un ejemplo pertinente es la aparición de 
nuevos valores, ligados a una reorganización conceptual de realidades existentes o novedosas. Un caso relevante puede ser el de la ética medio ambiental o ecológica. Se supone que la naturaleza constituye una base permanente y relativamente fija del entorno humano, social y moral. Y, sin embargo, los cambios radicales provocados por la acción del ser humano sobre ella han conducido a cambios en los dos aspectos mencionados por Tavani (2002). Por un lado, ha inducido un cambio en la conceptualización del entorno natural: ya no se piensa como el medio pasivo, inerte y fijo supuesto por las teorías éticas tradicionales, sino como una entidad que, a semejanza de los seres vivos, está sujeto a procesos de cambio, de degeneración o regeneración que, en la época postindustrial, tienen mucho que ver con la acción humana. A su vez, esa reconceptualización, esa nueva forma de ver el entorno natural, ha tenido como consecuencia la aparición de nuevos valores, si no absolutos, sí radicalmente nuevos en su aplicación a la naturaleza. La exigencia de conservación y de cuidado es un valor moral impensable cuando se trata de aplicarlo a una realidad inerte e inmodificable, pero adquiere sentido cuando atañe a una realidad que se equipara con un organismo vivo. En este caso, la reconceptualización de una realidad existente amplia nuestras teorías éticas no sólo en cuanto a su aplicación, sino en cuanto a su naturaleza, en la medida que hacen emerger nuevos valores en nuevos ámbitos. Ambas dimensiones, reconceptualización y reteorización, pueden y suelen ir estrechamente relacionadas.

Lo mismo se puede decir respecto a la dicotomía que establece Tavani en la interpretación en la interpretación de la tesis de la singularidad de la ética computacional. Según Tavani $(2002,41)$, los abogados de la novedad de la ética computacional han de decidirse o bien por la novedad de los objetos éticos que introduce en el ámbito del discurso, o por la novedad de las cuestiones éticas que se generan, como si se tratara de una disyuntiva excluyente. Es cierto que algunos teóricos (como Maner, 1996) han sostenido la originalidad y singularidad de las cuestiones éticas planteadas por las TIC, sin que por ello hayan admitido una modificación en su ontología moral (los sujetos u objetos éticos que reconoce), pero no es menos cierto que lo contrario aparenta ser más dificultoso. La admisión de nuevos objetos éticos lleva aparejada no solamente una extensión en la aplicación de valores tradicionales morales, sino también la aparición de valores emergentes. En general, una reacomodación radical del ámbito moral, que puede desplazar el objeto y el peso de la aplicación de los valores morales, así como el conjunto de categorías (sujeto moral, derechos y deberes morales, etc.) que tradicionalmente han formado parte de la filosofía moral.

Y, sin embargo, también es defendible la tesis, más moderada, de que, incluso admitiendo una ampliación de la ontología moral, adscribiendo a nuevos objetos la posibilidad de ser objeto de consideración moral, las teorías éticas tradicionales (utilitaristas, kantianas o neokantianas, virtuosas, etc.) son perfectamente capaces de dar cuenta y asimilar esa extensión del ámbito de lo moral. En definitiva, la cuestión sería entonces si esas teorías disponen de los 
recursos conceptuales e inferenciales para poder integrar las nuevas realidades morales. Una vez más, los ejemplos que se pueden invocar atañen a la ética animal y a la medio ambiental: del mismo modo que estas éticas han incorporado, en cierto sentido, nuevos objetos morales (como el resto de las especies animales o la naturaleza) sin necesidad de introducir grandes cambios en las teorías éticas disponibles, igualmente podría suceder lo mismo en la ética computacional. En cierto sentido, la cuestión metodológica, a la que se refiere Floridi (1999, 33), se puede reducir a un asunto de economía cognitiva (admitiendo un cierto principio de parsimonia teórica: no recurrir a más instancias ni principios explicativos de los estrictamente necesarios). Si las teorías éticas tradicionales nos permiten dar cuenta, en el sentido cognitivo, de las nuevas realidades morales relacionadas con las TIC, sean éstas nuevos objetos o valores emergentes, ¿por qué recurrir a una reorganización radical (ontológica, epistemológica, moral, y quizás estética) del reino de lo real?

\section{La infoética de L. Floridi}

L. Floridi (1999, 2002; Floridi y Sanders, 2001) ha propuesto una reconsideración radical de los problemas éticos ligados a las TIC. Tal reconsideración parte, por un lado, de la constatación de la insuficiencia de las teorías éticas tradicionales y, por otro, de la necesidad conceptual de desplazar el centro de la reflexión ética a la hora de analizar los problemas morales entrañados por las TIC. Ello le ha llevado a proponer una nueva teoría que, quizás por su carácter radical, aún no ha merecido la suficiente atención (cfr. no obstante Siponen, 2004). Tal teoría pretende separarse de las teorías éticas tradicionales en no estar orientadas a la acción humana, a su evaluación moral y al análisis de sus consecuencias prácticas. Según Floridi, el sesgo de las teorías éticas tradicionales (consecuencialismo, contractualismo y deontologismo) hacia la acción humana es la causa de una antropomorfización en la ética de los computadores. Ese carácter antropomórfico se concreta en la conceptualización de los artilugios tecnológicos (ordenadores, programas,....) como agentes morales, como sujetos de responsabilidades morales. Ello es así porque las teorías morales kantianas neokantianas están ideadas para contextos de relación humana y social, y no son capaces de integrar de una forma satisfactoria contextos éticos que no se ajustan a esa situación prototípica. En concreto, «los imperativos morales de Kant parecen tener dos problemas. Ni la ley de imparcialidad (la regla de Oro) ni la ley de universalidad (actúa como un legislador universa) son suficientes para afrontar:

1) los problemas de la ética de los computadores que no implican a seres humanos $[\ldots]$

2) problemas de la ética de los computadores de naturaleza lúdica» (Floridi, 1999, 40) 
Desde un punto de vista cognitivo, las deficiencias de las teorías éticas tradicionales se traducen en una imposibilidad de integrar conceptualmente los casos radiales o periféricos, en una incapacidad para extender el esquema categorizador en los casos que, aparentemente, no comparten su estructura con el caso prototípico. En ese caso, son perfectamente distinguibles los componentes de la situación: un agente moral (un ser humano), que realiza (o escoge realizar) una acción $\mathrm{A}$, que tiene consecuencias para un conjunto de pacientes $\mathrm{P}$ que también son, en el caso paradigmático, otros seres humanos. Ahora bien, Floridi (1999) llama la atención sobre el hecho de que ciertas situaciones en las que intervienen las TIC no se ajustan a ese esquema conceptual. El efecto general de esas situaciones es de diluir: 1) la naturaleza del agente moral, en la medida en que puede tratarse de un objeto no humano (un programa, un sistema...), o un sujeto distribuido (un conjunto de individuos, o una mezcla de individuos y artilugios tecnológicos); 2) la naturaleza de la acción moral efectuada; o bien porque su carácter complejo difumine sus contornos de definición, o bien porque algunas de sus notas características (su inmaterialidad, su naturaleza prácticamente anónima, etc.) atenúan la percepción de la acción en cuestión para los que participan en ella. Así, en muchas ocasiones no sólo la acción misma, sino su presunta relación con un conjunto de pacientes resulta distorsionada al no podérsele aplicar la plantilla (la imagen esquemática) de una situación prototípica. Ni se puede definir claramente el sujeto de la acción y, por tanto, adscribirle una responsabilidad moral, ni la acción misma es localizable, ubicable o atribuible a un sujeto, ni resultan claras las consecuencias para un conjunto indefinido de pacientes.

Ante estas deficiencias en la aplicación de teorías clásicas éticas, Floridi (1999) buscó inspiración en éticas no clásicas, no solamente en el sentido de abrir nuevos campos de reflexión y aplicación de las teorías éticas, sino de teorías que hubieran modificado en algún sentido interesante la distribución de los focos teóricos de la acción moral, la triada sujeto/acción/objeto.

Las candidatas inmediatas eran la ética médica, la bioética y la ética medioambiental o ecológica. Lo que tenían en común estas nuevas teorías éticas eran dos cosas: 1) una ampliación de los horizontes de la ética, en el sentido de que, cada una, extendía el ámbito de la reflexión moral a nuevas realidades; y 2) un desplazamiento del centro de la reflexión ética, del sujeto moral al paciente moral. En el caso de la ética médica, el peso de la discusión se traslada de la responsabilidad del médico (sus decisiones) a los derechos del paciente (a ser informado, a decidir sobre su futuro, etc.) En el caso de la bioética, surgieron las cuestiones que tienen que ver con la integridad de la vida biológica y su preservación, desde los presuntos derechos del feto a la manipulación genética, pasando por todas las cuestiones suscitadas por la ética animal (los derechos de las especies animales no humanas). Y, finalmente, en lo que se refiere a la ética medioambiental, se procede a una extensión considerable del concepto de paciente moral: no sólo los seres biológicos son considerados como titulares de derechos morales, sino que tales derechos se extienden también a los seres inanimados, 
como entornos o sistemas ecológicos. Según Floridi: «Al capturar lo que es preteórico, pero una intuición muy extendida, compartida por la mayoría de la gente, mantienen la tesis general de que cualquier forma de vida tiene algunas propiedades esenciales o intereses morales que merecen y requieren ser respetados. Argumentan que la naturaleza y el bienestar del paciente de una acción constituyen su posición moral y que éste realiza exigencias vitales en el agente que con él interactúa y debe contribuir a guiar sus decisiones éticas y a delimitar su conducta moral» (Floridi, 1999, 42).

Más allá de la discusión de si éste es un movimiento aceptable para la teoría ética, es conveniente advertir la naturaleza del recurso conceptual o cognitivo, que no es otro que el de la recategorización o reconceptualización de los individuos en el universo del discurso moral. Todas esas teorías éticas no estándares proceden mediante una ampliación del universo del discurso, que tiene dos consecuencias principales:

1) una ampliación de la clase de objetos que son admitidos en la ontología del universo. En el caso de la bioética, la clase de los seres vivos animados y, en el de la ética medioambiental, las complejas estructuras formadas por seres animados o inanimados.

2) una proyección de la estructura relacional del dominio prototípico original del discurso moral al nuevo universo, poblado de nuevas realidades. Aunque es cierto que el peso o la perspectiva bajo la que se considera ese universo es diferente, porque el paciente moral adquiere la principal relevancia, la triada sujeto/acción/objeto permanece inalterada.

Para ser más explícito, el recurso cognitivo utilizado no es otro que el de la proyección analógica o metafórica (Lakoff, 1993; Lakoff y Johnson, 1999) que traslada, por una parte, el estatuto ontológico -o, en este caso, moral- de los objetos del discurso y, por otra, la estructura relacional que articula esos objetos en el dominio fuente. La condición estructural esencial que posibilita la operación cognitiva es el principio o hipótesis de invarianza (Lakoff, 1990) que asegura la preservación de la topología cognitiva del dominio fuente. El respeto al principio, que tiene un carácter local (no hay estructuras cognitivas globales o exclusivas de un dominio fuente), permite caracterizar la clase de las proyecciones metafóricas que son admisibles de un determinado ámbito, esto es, posibilitan distinguir entre las proyecciones metafóricas que son epistémicamente legítimas de las que son simples errores categoriales. No es éste el lugar para desarrollar en detalle las características formales de las operaciones de proyección ni para analizar exhaustivamente la naturaleza de sus resultados, la configuración de espacios mentales o espacios de fusión conceptual (blended spaces, Fauconnier, 1997), pero sí que resulta útil examinar las propuestas de la ética de la información a la luz de ese marco conceptual que proporcionan las ciencias cognitivas. 
Basándose en el tipo de movimientos conceptuales efectuados por las éticas no clásicas, Floridi (2002) formula dos exigencias:

1) es preciso ampliar el ámbito de los agentes y pacientes morales para que, entre ellos, se incluyan no sólo los congéneres del sujeto moral prototípico (el ser humano), sino todo tipo de objetos; en general, los objetos informacionales u objetos de datos: «La primera tesis formula que los objetos de información, en cuanto tales objetos de información, pueden ser agentes morales. Esto significa no sólo que se analice a un $a$ interpretado como un objeto de información, sino mostrando más bien que $a$ puede ser interpretado correctamente como un objeto de información (esto es, que un agente artificial, como un elemento de software puede desempeñar un papel de agente moral) en el normal nivel de abstracción que adoptan otras teorías éticas» (Floridi, 2002, 290).

¿Cuál es el fundamento cognitivo de esa ampliación del universo del discurso moral? De acuerdo con las teorías éticas tradicionales o estándares, el carácter moral de los agentes está intrínsecamente unido a su libertad, en última instancia a su condición de seres intencionales. Esa característica del sujeto moral se pierde en el universo moral propuesto por la ética de la información.

2) Pero Floridi no se limita a proponer un modo de análisis, un cierto nivel de descripción (correspondiente a lo que él denomina nivel de abstracción), sino que defiende una recategorización estricta (una interpretación) de los sujetos morales. El fundamento de esa reconceptualización no es que los objetos de información sean libres o intencionales, sino que tengan un valor moral: «La segunda tesis afirma que los objetos de información, en cuanto tales, pueden tener un valor moral intrínseco, aunque posiblemente de mínima cuantía y, en consecuencia, pueden ser pacientes morales, sujetos de un grado de respeto moral, igualmente mínimo» (Floridi, 2002, 290). Para justificar esta ampliación, Floridi (2002) maneja una variedad de argumentos que van desde el análisis crítico de las teorías kantianas o neokantianas a los propiamente metodológicos. El sentido general de sus críticas a las teorías éticas tradicionales es que son incapaces de hacer frente a las nuevas cuestiones morales planteadas por las TIC, de lo cual es buena muestra, en su opinión, las limitaciones de la ética de los computadores cuando se concibe en ese marco tradicional, como ética aplicada. Es decir, en este punto representa una posición derivada del debate de la singularidad de la ética de los computadores. Esa singularidad es la que provoca y hace necesario, el replanteamiento conceptual que introduce la infoética. Sólo desde este nuevo marco conceptual queda patente la singularidad de la ética de los computadores, y sólo con los instrumentos analíticos provistos por la infoética es posible dar un tratamiento comprehensivo y coherente de la ética de los computadores. 
Otros argumentos utilizados por Floridi (2002) parecen menos cogentes o convincentes. Por ejemplo, el que parece incurrir en una falacia de dominó (slippery slope). Floridi parte de la constatación de que el desarrollo de la teoría ética ha supuesto la progresiva ampliación del universo del discurso moral, desde un punto de partida kantiano, antropocéntrico. Así, la ética medioambiental o ecológica ya no es antropocéntrica, sino biocéntrica, porque considera que todos los seres vivos son o pueden ser sujetos morales y, en todo caso, objetos de respeto moral. La ética ecológica se fundamenta pues en la proyección analógica de un dominio fuente (source domain) tradicional, el que sitúa el ámbito de lo moral en la relación entre los individuos y la sociedad en la que viven, en un dominio diana (target domain), que es el de la biosfera:

\begin{tabular}{lll}
\hline Sociedad & & Biosfera \\
\hline Individuos & $\longrightarrow$ & Seres vivos \\
\hline Ciudadanos & $\longrightarrow$ & Sistemas biológicos \\
\hline
\end{tabular}

El efecto que provoca esta proyección analógica es una conceptualización nueva: los seres vivos son comprendidos, en su relación con la biosfera, del mismo que los seres humanos en relación con la sociedad: los seres vivos son ciudadanos de la biosfera. En cuanto tales, pueden ser titulares de derechos y deberes morales de modo similar a los que tradicionalmente se asignan a los ciudadanos.

Ahora bien, razona Floridi $(2002,291)$, ¿por qué detenerse en el nivel - de abstracción - que supone la vida biológica? ¿en virtud de qué razonamiento los seres vivos constituyen un nivel óntico superior al de los seres vivos?: «Si los seres humanos corrientes no son las únicas entidades que disfrutan de alguna forma de respeto moral, ¿qué más lo tiene? ¿sólo los seres sensible? ¿sólo los sistemas biológicos? ¿Qué justifica incluir algunas entidades y excluir otras? Supóngase que reemplazamos una concepción antropocéntrica por otra biocéntrica, ¿por qué el biocentrismo y no el ontocentrismo?» (Floridi, 2002, 291). Precisamente lo que propone Floridi es optar por el ontocentrismo, que supone el máximo nivel de abstracción/descripción de la realidad, a la hora de elaborar una teoría ética fundamentadora (una macroética) de las éticas particulares, como la ética de los computadores. Por tanto, lo que propone es prolongar la proyección metafórica en el siguiente sentido:

\begin{tabular}{|c|c|c|}
\hline Antropocentrismo & Biocentrismo & Ontocentrismo \\
\hline Individuos & Seres vivos & Objetos de información \\
\hline Ciudadanos & Sistemas biológicos & \\
\hline Sociedad & Biosfera & Infosfera \\
\hline
\end{tabular}

Ahora bien, el problema para la infoética de Floridi es que las proyecciones metafóricas no pueden ser completas, que las estructuras relacionales que caracterizan cada uno de los dominios no se pueden trasladar sin más de uno a otro. 
Por poner el ejemplo más evidente, que afecta no sólo a la infoética sino también a las éticas medioambientales, en la ética antropocéntrica (humanista) se da una homogeneidad en los miembros del universo que no existe en las otras. Tal homogeneidad consiste en que todos los miembros califican como agentes o pacientes morales, es decir, que las relaciones que desde un punto de vista moral se suscitan entre ellos tienen un carácter simétrico. Además, esa homogeneidad posibilita la emergencia de nociones morales, como el autorespeto, que sencillamente carecen de sentido cuando se amplian las ontologías morales, cuando se reconoce como moralmente significativos a otros individuos. En las éticas informacionales y ecológicas se da una homogeneidad ontológica, pero no moral: los seres vivos y los objetos de información pertenecen a una misma clase óntica, pero a diferentes conjuntos de individuos morales, agentes y pacientes. De tal modo que, por decirlo así, lo que se proyecta a esas éticas no clásicas es una categorización moral que divide al universo del discurso moral en subconjuntos no homogéneos. Por un lado, los agentes morales que, por su naturaleza de agentes son por ello mismo susceptibles de ser pacientes morales y, por otra, los pacientes morales. En el caso de la ética clásica los pacientes morales son también, por definición, posibles agentes morales. En cambio, en el caso de las éticas no clásicas los pacientes morales pueden ser sólo eso, objetos de una acción moral, y estar en una relación asimétrica con respecto a los agentes morales.

El problema es entonces definir en qué consiste ser un paciente moral, qué convierte a un objeto de información, como un programa informático, en algo que desempeña un lugar o posición dentro de una teoría macroética: «Una vez que se restringe el análisis al caso en que $\mathrm{x}=\mathrm{p}$ (aciente), la cuestión que hay que abordar es: ¿existe algún grado de valor intrínseco y no condicionado (esto es, ni instrumental ni emocional) en la naturaleza de un $\mathrm{p}=$ objeto de información tal que deba determinar, restringir, guiar o conformar las acciones morales de un a(gente)? Esto es, ¿tiene un objeto de información como $p$ un valor moral intrínseco tal que, ceteris paribus, puede contribuir a la configuración moral de la conducta de $a$ ?» (Floridi, 2002, 292). La respuesta de Floridi es que todos los objetos de información pueden ser pacientes morales en virtud de su valor intrínseco.

Su análisis procede a partir de una crítica de la axiología kantiana, pero, como no merece la pena comentar los detalles de esa crítica, sólo mencionaremos su conclusión general: en cada uno de los niveles ontológicos (o de descripción) de la realidad, los objetos que constituyen o son reconocidos en ese nivel tienen un valor intrínseco. Así, un ser humano despojado de algunas (o de todas) las características que le convierten en ser humano sigue teniendo un valor intrínseco en cuanto ser vivo, o sistema biológico, es decir, en cuanto parte de un nivel superior de abstracción o descripción de la realidad.

El nivel más general de abstracción/descripción de la realidad es el que conceptualiza a los elementos de la realidad como objetos de información. Pues en ese nivel tan general los objetos de información son acreedores de respeto moral, porque también en ese nivel pueden ser objetos de acciones morales. 
Aunque Floridi apela a un ejemplo engañoso ${ }^{1}$, sirve éste para ilustrar su concepción jerárquica de lo que es el valor intrínseco y el correspondiente respeto moral: «Como veremos, una entidad x puede ser respetada en diferentes niveles de abstracción, incluyendo el nivel en que x sólo es un objeto de información. Así, por ejemplo, en el caso de María, la ética de la información sostiene que:

C) si María califica como organismo vivo, entonces se aplican las consideraciones éticas biocéntricas. Sin embargo, supóngase que María ya no califica como organismo vivo. Su cadáver disfruta todavía de un grado de valor moral intrínseco por su naturaleza como objeto de información y, como tal, aún mantiene la correspondiente exigencia de respeto moral» (Floridi, 2002, 296).

Una de las críticas inmediatas a esta posición de Floridi es la de panmoralismo: si todo componente de la realidad puede ser descrito como un objeto de información, y tiene un valor intrínseco, entonces todo lo real merece un respeto moral y puede ser paciente de una acción moral. O, para ponerlo en términos negativos, ¿qué es lo que no es un objeto de información y, por tanto, no merece una consideración moral? La definición de objeto de información es tan general, tan omnicomprensiva que, en realidad, sólo los objetos ilógicos (inconsistentes) no son objetos informacionales. Como bien señala Floridi: «El único sentido significativo en que es posible hablar de 'algo' que no alcanza a figurar como una entidad informacional es hablar de un objeto que sea intrínsecamente imposible, por ejemplo, una contradicción lógica en sí misma. Existe un número infinito de objetos inconsistentes, pero como cualquier cosa puede ser predicada de todo objeto inconsistente, sólo existe un tipo de objeto que pueda ser intrínsecamente carente de valor y no respetable. Llamémosle C. C representa el grado cero en nuestra escala de valor moral» (Floridi, 2002, 300-01). He aquí una curiosa conjugación de consideraciones lógicas y morales: en realidad sólo existe un (tipo de) objeto que carece de valor intrínseco y ése es la C(ontradicción), porque todas las contradicciones son equivalentes entre sí, en cuanto a su naturaleza informacional. Ciertamente, es una forma original de ver la lógica, pero de la concepción de Floridi se sigue no sólo que las contradicciones están mal, sino que son el mal. Una contradicción encierra en sí el grado máximo de entropía, de una entropía que no es graduable, sino en cierto modo absoluta y, por ello, también constituye el escalón más bajo del ámbito de lo moral.

Por otro lado, consideremos las (¿nefastas?) consecuencias que, en su aplicación práctica a los seres humanos, podría tener esta concepción, si las consideramos en el nivel de abstracción que propone Floridi, el de objetos de información. En cuanto tales, los seres humanos pueden equipararse a sus sistemas de creencias en

${ }^{1}$ El ejemplo aducido por Floridi, el de que un objeto, como un cadáver, sigue siendo acreedor a un respeto moral es engañoso porque la profanación no es una falta de respeto moral que se ejerza sobre un objeto en cuanto tal objeto de información, sino en cuanto a su valor simbólico que, como en otros casos de simbolismo (por ejemplo, la profanación de una bandera), se fundamenta cognitivamente en mecanismos como la metonimia. En ese sentido, la cremación, moralmente correcta, puede sin embargo ser considerada como una falta de respeto moral a un cadáver, en cuanto objeto de información. 
términos naturalistas ( si uno es dualista, a sus sistemas de creencias y a algo diferente, su ser 'material') -en la medida en que esas creencias están 'incorporadas' en su configuración neuronal, de tal modo que, en ese nivel, los humanos serían moralmente respetables en la medida en que lo fueran sus sistemas de creencias. Ahora bien, si no es uno un soberbio epistémico (una persona que está seguro de que entre sus creencias no se da ninguna inconsistencia) y, de una manera humilde, admite que seguramente, bien en un nivel local o en uno global, el conjunto de creencias que sostiene es inconsistente, entonces su valor moral caerá al nivel de $\mathrm{C}$, al nivel más bajo de la consideración moral, la que merece el mal absoluto.

Para hacer justicia a la concepción de Floridi es necesario, no obstante, tener en cuenta las siguientes dos consideraciones:

1) Existe una relación jerárquica entre los niveles en que es aplicable la noción de valor (y respeto) moral. El nivel más abstracto (el de los objetos de información) determina un nivel mínimo de apreciación moral. Quizás lo que sucede es que, vistos desde ese nivel, los seres humanos (nuestros sistemas de creencias) seamos mínimamente apreciables. Pero ese nivel de abstracción /descripción es subordinado a otros, que priman (override) sobre el nivel general. Por ejemplo, nuestra naturaleza de seres vivos, por no decir nuestras kantianas propiedades de seres conscientes, intencionales y libres.

2) Por otro lado, no sólo los seres humanos, en cuanto objetos de información, son moralmente (in)calificables. También lo son sus acciones, que son un subconjunto de los objetos de información y que, en la terminología informacional de Floridi, son caracterizadas como mensajes. Los mensajes no son sólo objetos de información sino que, además, son procesos que pueden afectar a otros objetos de información, de forma positiva o negativa. En general, los mensajes negativos se corresponden con acciones moralmente malas, en la medida en que afectan a la integridad informacional de un paciente, esto es, aumentan de una forma u otra su entropía. En el caso límite, cuando atentan a la propia existencia del paciente moral, los mensajes (las acciones) son malas en extremo: «En términos más metafísicos, cualquier proceso que niegue la existencia, en la medida en que existe, no merece respeto (nótese que aún puede merecer respeto por otras razones, que primen), pero cualquier cosa que exista, en la medida en que existe, merece un cierto respeto, en cuanto entidad [...] Desde la perspectiva de la programación orientada a objetos, sólo puede existir el mal en términos de mensajes negativos, esto es, acciones moralmente malas. Estas acciones son intrínsecamente merecedoras de falta de respeto, y no han de ser causadas, sino prevenidas, eliminadas o modificadas, de tal modo que dejen de ser malas» (Floridi, 2002, 301).

La concepción de la ética de la información acaba pues en una exaltación de la existencia, que es moralmente preferible a la no existencia. Todo objeto, en 
cuanto objeto de información, merece una consideración moral. Esa consideración moral (respeto) se traduce en la obligación, para los agentes morales, de no atentar contra la naturaleza informacional del objeto, en particular contra su existencia. A primera vista, parecería que se trata de una concepción inviable, en el sentido de que imposibilitaría, entre otras cosas, la eliminación de animales o plantas (o minerales) para el consumo humano. Esto sería así, si no fuera porque los principios morales de la ética de la información, como los de otras teorías éticas, se aplican de acuerdo con cláusulas ceteris paribus, esto es, suponiendo la no aplicabilidad de principios éticos de orden superior. Es de suponer que la destrucción de objetos de información con fines alimenticios esté justificada por esa clase de principios...La ética de la información es una ética que se aplica en el máximo nivel de abstracción, esto es, cuando no se aplican niveles más bajos de abstracción o, lo que es lo mismo, niveles más finos de descripción. Por eso se ha puesto en cuestión su carácter práctico (Siponen, 2004): podría darse el caso de que, aunque correcta, la ética de la información fuera vacua porque, de hecho, no se dieran nunca condiciones para su aplicabilidad, porque sus principios siempre serían postergados a favor de los que operan en un nivel más bajo de abstracción.

\section{Conclusiones}

Se supone que la infoética es una ética no humanista, en el sentido de ser una ética no antropocéntrica, de no centrar en el individuo los valores morales. Pero, así concebida, la primera crítica que se puede hacer a tal ética tiene poco que decir respectos a las acciones morales concebidas en términos tradicionales, prototípicas: a) los objetos informacionales pueden ser pacientes morales, es decir, objeto de acciones morales pero, es de suponer, sólo un subconjunto de los objetos informacionales pueden ser sujeto, esto es, agentes, de acciones morales. Se supone que ese subconjunto es el formado precisamente por los seres humanos, aunque es posible que un sujeto distribuido (individuo+artefactos tecnológicos) pueda ser considerado también un agente moral. En cuanto tal agente moral, no obstante, la responsabilidad de sus acciones ha de recaer en la parte intencional de ese sujeto distribuido. Dicho de otro modo, las acciones de un sujeto pueden estar distribuidas, pero no su responsabilidad moral.

b) Si se equipara la noción de mal, y el correspondiente deber moral de evitarlo -no causarlo conscientemente - con alguna noción de entropía, tenemos dos consecuencias en cuanto a la división de la infosfera en agentes y pacientes morales:

1) los pacientes morales pueden causar mal o, mejor, no todos pueden ser responsabilizados por la existencia de un mal moral (entropía). Los pacientes morales sin conciencia, esto es, incapaces de actuar conscientemente, no 
pueden causar un mal moral, aunque puedan ser la causa de la introducción de entropía en la infosfera: en términos de Floridi y Sanders (2001) pueden causar un mal natural. A pesar de lo que mantienen dichos autores (Floridi Sanders, 2001, 59-60), la existencia de un agente artificial no parece suficiente ni para dotar a una acción de una dimensión moral, ni para distinguir una noción intermedia de mal que no es moral ni natural, sino artificial. Sólo los seres humanos conscientes pueden voluntaria e intencionalmente causar el tipo de mal propiamente moral, y que tal causación les sea imputable, les sea adscribible una responsabilidad.

2) Aunque los únicos posibles responsables de un mal moral en la infosfera sean los seres humanos conscientes, no todo mal en ese ámbito les es imputable. De hecho la propia noción de mal moral, referido a los que pueden causarlo, es relativa. Un ser humano puede contribuir a aumentar la entropía en la infosfera sin saberlo porque, por ejemplo, desconozca el estado entrópico previo, o todas las consecuencias de su acción, etc. En ese sentido, no se puede definir una noción absoluta de mal -tal como parece implicar la noción de entropía -, sino sólo una noción relativa al conocimiento del sujeto moral. Como en cualquier otra teoría ética (micro o macro), la responsabilidad moral tiene como condición de posibilidad la conciencia y la intencionalidad. Sólo un ser consciente, por tanto, podría ser considerado responsable de cualquier alteración en la entropía ejercida por su mano.

En las teorías éticas clásicas, teorías centradas en el sujeto moral, según Floridi, la normatividad moral adopta una doble forma, la de derechos y la de deberes. En la infoética que propone Floridi que pretende desplazar el centro de la ética al objeto informacional, tal doble dimensión queda alterada. Todos los objetos informacionales, por el hecho de serlo, son titulares de derechos, aunque sea mínimos; son dignos, por decirlo como Floridi, de un respeto moral, puesto que como tales objetos, tienen un valor intrínseco. Ese valor es un valor óntico, por decirlo así: se trata del derecho a ser y, en todo caso, a no ver disminuida su naturaleza informacional, a no ver aumentada su entropía. Ahora bien, tales 'derechos' no generan deberes para cualesquiera objetos informacionales. Solamente los seres humanos son capaces de representarse los objetos informacionales, incluidos ellos mismos, en cuanto acreedores de esos derechos y, por tanto, sólo para ellos esos derechos son fuente de normatividad. La pérdida de la estructura relacional del dominio de base, a partir del cual se efectúan las sucesivas ampliaciones del ámbito del discurso moral, es la causa de que desaparezcan en esas expansiones nociones relevantes desde el punto de vista moral. Y eso vale tanto para la ética medioambiental como, en el nivel máximo de abstracción, para la ética de la información que propone L. Floridi. 


\section{REFERENCIAS BIBLIOGRÁFICAS}

FAuConnier, G. (1997), Mappings in Thought and Language, Cambridge: Cambridge U. Press.

FLORIDI, L. (1999), «Information ethics: on the philosophical foundation of computer ethics», Ethics and Information Technology, 1, 1: 37-56.

- (2002), «On the intrinsic value of information objects and the infosphere», Ethics and Information Technology, 4: 287-304.

FLORIDI, L, y J. W. Sanders (2001), «Artificial evil and the foundations of computer ethics», Ethics and Information Technology, 3: 55-66.

LAKOFF, G. (1990), «The invariance hypothesis: Is abstract reason based on image schemas?, Cognitive Linguistics, 1. 39-74.

- (1993), «The Contemporary Theory of Metaphor», en A. Ortony, ed., Metaphor and Thought, $2^{\mathrm{a}}$ ed., Cambridge: Cambridge U. Press, 202-251.

Lakoff, G. y M. Johnson (1999), Philosophy in the Flesh, Chicago: Chicago U. Press.

MANER, W. (1996), «Unique ethical problems in information technology», Science and Engineering Ethics, 2, 2: 137-154.

SIPONEN, M. (2004), «A pragmatic evaluation of the theory of information ethics», Ethics and Information Technology, 6: 279-290.

TAVANI, H. (2002), «The uniqueness debate in computer ethics: What exactly is at issue, and why does it matter?», Ethics and Information Technology, 4, 1: 37-54. 